\title{
Long-Term Risk of Stroke and Poststroke Outcomes in Patients with Heart Failure: Two Nationwide Studies
}

This article was published in the following Dove Press journal: Clinical Epidemiology

\begin{abstract}
Ying-Hsuan Tai ${ }^{1,2, *}$
Chuen-Chau Chang (iD) $2-4$

Chun-Chieh Yeh ${ }^{5,6}$

Li-Chin Sung iD ${ }^{7}$

Chaur-Jong $\mathrm{Hu}^{8}$

Yih-Giun Cherng (iD ${ }^{1,2}$

Ta-Liang Chen ${ }^{2,4,9, *}$

Chien-Chang Liao (iD) $2-4,10,11$

'Department of Anesthesiology, Shuang Ho Hospital, Taipei Medical University, New Taipei City, Taiwan; ${ }^{2}$ Department of Anesthesiology, School of Medicine, College of Medicine, Taipei Medical University, Taipei, Taiwan; ${ }^{3}$ Department of Anesthesiology, Taipei Medical University Hospital, Taipei, Taiwan; ${ }^{4}$ Anesthesiology and Health Policy Research Center, Taipei Medical University Hospital, Taipei, Taiwan; ${ }^{5}$ Department of Surgery, China Medical University Hospital, Taichung, Taiwan; ${ }^{6}$ Department of Surgery, University of Illinois, Chicago, IL, USA; ${ }^{7}$ Division of Cardiology, Department of Internal Medicine, Shuang Ho Hospital, Taipei Medical University, New Taipei City, Taiwan; ${ }^{8}$ Department of Neurology, Shuang Ho Hospital, Taipei Medical University, New Taipei City, Taiwan; 'Department of Anesthesiology, Wan Fang Hospital, Taipei Medical University, Taipei, Taiwan; ${ }^{10}$ Research Center of Big Data and Meta-Analysis, Wan Fang Hospital, Taipei Medical University, Taipei, Taiwan; ' 'School of Chinese Medicine, College of Chinese Medicine, China Medical University, Taichung, Taiwan

*These authors contributed equally to this work
\end{abstract}

Correspondence: Chien-Chang Liao Department of Anesthesiology, Taipei Medical University Hospital, 252 Wuxing Street, Taipei I 10, Taiwan

Email jacky48863027@yahoo.com.tw

Yih-Giun Cherng

Department of Anesthesiology, Shuang Ho Hospital, New Taipei City, Taiwan

Email stainless@s.tmu.edu.tw
Objective: To evaluate the long-term risk of stroke and poststroke adverse outcomes in patients with heart failure (HF).

Methods: We used research data from Taiwan's National Health Insurance Program from 2000 to 2005 and identified 20,072 adults aged $\geq 30$ years who were newly diagnosed with HF. Frequency matching based on age and sex was used to select a comparison cohort consisting of 80,288 adults without HF. Events of incident stroke were identified from medical claims during the 2000-2013 follow-up period. The adjusted hazard ratios (HRs) and $95 \%$ confidence intervals (CIs) of the association of stroke with HF were calculated with a multiple Cox proportional hazard model. Another nested stroke cohort study of 480,604 hospitalized stroke patients determined the adjusted odds ratios (ORs) and 95\% CIs for adverse events after stroke in patients with and without HF between 2000 and 2009.

Results: Compared with the non-HF cohort, HF patients had an increased risk of stroke (HR $2.32,95 \%$ CI 2.21-2.43), including ischemic stroke and hemorrhagic stroke. The association between $\mathrm{HF}$ and stroke was significant in both sexes and in patients in all age groups and with various medical conditions. Previous HF was associated with poststroke mortality (OR 1.40, 95\% CI 1.31-1.50), pneumonia (OR 1.33, 95\% CI 1.28-1.38), and septicemia (OR $1.30,95 \%$ CI 1.23-1.37).

Conclusion: HF was associated with a higher risk of stroke, and patients with HF had more complications and greater mortality after stroke.

Keywords: heart failure, adverse outcome, risk, stroke

\section{Introduction}

The latest global estimate shows that stroke remains a leading cause of death and disability and substantially contributes to the economic costs of healthcare systems worldwide. ${ }^{1}$ To reduce the burden of stroke, the management of common modifiable risk factors is of the utmost importance. Established modifiable risk factors of stroke include the consumption of alcohol and cigarettes, physical inactivity, hypertension, diabetes, dyslipidemia, and atrial fibrillation. ${ }^{2}$ To improve the management of stroke and its sequelae, it is essential to discover other potential risk factors and control them adequately.

Heart failure (HF) is also an important cause of a current global epidemic, affecting approximately 25 million individuals worldwide and imposing a huge economic burden that has been estimated to be greater than $\$ 100$ billion per annum. ${ }^{3,4}$ HF causes thromboembolism, hypoperfusion, and atherosclerosis and 
contributes to the pathological mechanisms of stroke. ${ }^{5}$ According to epidemiologic data, $10 \%$ to $24 \%$ of stroke patients suffer from comorbid chronic $\mathrm{HF}^{5}$

The risk of stroke in patients with HF has been evaluated in previous studies. ${ }^{6-17}$ However, the relationship between $\mathrm{HF}$ and stroke risk is not completely understood because of controversial findings of previous studies. ${ }^{7,11,12,14}$ Some studies indicated the higher risk of stroke persisted over time in HF patients, ${ }^{7,12,14}$ but another study reported the stroke risk was normalized 6 months after HF diagnosis. ${ }^{11}$ Moreover, there were two studies showed an increased risk of hemorrhagic stroke in HF patients, ${ }^{12,14}$ while another one study failed to investigate the association between HF and risk of hemorrhagic stroke. ${ }^{11}$ There were some study limitations in the previous studies, such as small sample size of patients with $\mathrm{HF}(<10,000 \mathrm{HF}$ subjects $){ }^{6-13,15-17}$ inadequate follow-up period (<10 years), ${ }^{6-11,13,16,17}$ lack of consideration of atrial fibrillation, ${ }^{10-12} \mathrm{HF}$ severity, ${ }^{11,12,14,15}$ and type of stroke. ${ }^{6-10,13,15-17}$ In addition, most studies do not examine the impact of HF on hospitalized stroke patients in terms of important clinical outcomes. ${ }^{6-11,13-17}$ Accordingly, we conducted two nationwide studies to evaluate the long-term risk of stroke in patients with HF and the effects of a history of HF on the outcomes of admitted stroke patients.

\section{Methods}

\section{Source of Data}

We conducted this study by utilizing reimbursement claims submitted to Taiwan's National Health Insurance Program, which was implemented in March 1995. This universal insurance program covers more than $99 \%$ of Taiwanese and non-Taiwanese working or studying in Taiwan. A detailed description of the research database was given in our previous studies. ${ }^{18,19}$ Informed consent was not required because the analysis used preexisting deidentified data. This study was approved by the institutional review board of Taipei Medical University (TMUJIRB-202010041; TMU-JIRB-201705065; TMU-JIRB201705063).

\section{Study Design}

In a longitudinal cohort of one million insured individuals (Study I), we identified a HF cohort of 20,072 patients aged more than 30 years with HF that was diagnosed between 2000 and 2005 who did not have a previous record of $\mathrm{HF}$ diagnosis or treatment in the database, which was established in 1996, or a history of stroke before the index date. To confirm that the patients had HF with more certainly, this study required that at least two visits for outpatient medical services (due to a physician's principal diagnosis of HF) or at least one visit for inpatient care due to $\mathrm{HF}$ had occurred. Using frequency matching (case-control ratio 1:4) by age and sex during the same index period, we identified a non-HF cohort of 80,288 patients who had no history of HF or stroke before the index date. To ensure that both cohorts were free of stroke at the start of the study, patients with any diagnosis of stroke within previous four years before the first diagnosis of HF were excluded from this analysis. The occurrence of stroke was identified during the followup period from 1 January 2000 to 31 December 2013. Our purpose was to evaluate the risk of stroke in patients with $\mathrm{HF}$ in this retrospective cohort study. Because ageing and incident comorbidities for the association between HF and stroke risk during the follow-up period, we considered the delta change of CHA2DS2-VASc score as one of covariates in this study.

In Study II, we conducted a nested cohort study including 480,604 patients aged $\geq 30$ years who were hospitalized because of stroke between 2002 and 2009, and 30,532 of these patients had a history of HF. We compared the 30-day poststroke adverse outcomes, including pneumonia, septicemia, urinary tract infection, intensive care and mortality, in hospitalized stroke patients with and without a history of HF. The utilization of medical resources during stroke hospitalization was also compared between patients with and without HF, including the length of the hospital stay and medical expenditures.

\section{Measures and Definitions}

We defined the income status of each study subject according to the definition of the Ministry of Health and Welfare, Taiwan. Physicians diagnostic codes from the International Classification of Diseases, Ninth Revision, Clinical Modification were used to identify diseases and morbidities, including hypertension (ICD-9-CM 401-405), chronic obstructive pulmonary disease (ICD-9-CM 491, 492, 496), mental disorders (ICD-9-CM 290-319), diabetes (ICD-9-CM 250), atherosclerosis (ICD-9-CM 440), hyperlipidemia $(272.0,272.1,272.2)$, liver cirrhosis (ICD9-CM 571), atrial fibrillation (ICD-9-CM 427.31), and Parkinson's disease (ICD-9-CM 332). Hemodialysis (D8) and peritoneal dialysis (D9) were also recorded according to the administrative code of Taiwan's National Health Insurance Program. Medications (statins and metformin) 
and previous medical visits to emergency care and inpatient care were identified from the records of medical facilities. Thirty-day in-hospital mortality and infectious complications during or after the index stroke admission were the outcomes of the study. The infectious complications included pneumonia (ICD-9-CM 480-486), septicemia (ICD-9-CM 038 and 998.5), and urinary tract infection (ICD-9-CM 599.0).

In Taiwan, physicians considered medical history and findings of physical examination, chest radiography and echocardiography to identify patients with HF in the clinical settings. ${ }^{20}$ Framingham clinical criteria was also applied for the diagnosis of $\mathrm{HF}$ that is the concurrent presence of either two major criteria or one major criterion and two minor criteria based on clinical manifestations. ${ }^{21}$ Echocardiography is used to differentiate HF with reduced or preserved ejection fraction. ${ }^{20}$ B-type natriuretic peptide or N-terminal proB-type natriuretic peptide are measured to assist in confirming or excluding the diagnosis of heart failure. $^{20}$ In this study, we considered patients with HF who had at least two visits for outpatient medical services (due to a physician's principal diagnosis of HF) or at least one visit for inpatient care due to HF had occurred.

In Study II, we considered the use of antibiotics included penicillins (such as amoxicillin, amoxicillinclavulanate, and ampicillin-sulbactam), first-generation cephalosporins (such as cephalexin, cefadroxil, cefazolin, and cephradine), second-generation cephalosporins (such as cefuroxime), macrolides (such as azithromycin and clarithromycin), quinolones (such as ciprofloxacin and levofloxacin), and sulfonamides (such as trimethoprim and trimethoprim-sulfamethoxazole) within 3 months before stroke admission.

\section{Statistical Analysis}

In Study I, we used chi-square tests to examine the distributions of the categorical data, including age, sex, income status, history of diseases, and visits to emergency care and inpatient care, in the HF cohort and the non-HF cohort. The adjusted hazard ratios (HRs) with 95\% confidence intervals (CIs) of stroke associated with HF were calculated using multiple Cox proportional hazards models. Subgroup analysis was also performed to determine the association between $\mathrm{HF}$ and stroke risk using multiple Cox proportional hazards models. We also determined the joint effects of $\mathrm{HF}$ and coexisting medical conditions on the risk of stroke, which are described in the supplementary files. The association between stroke and component of CHA2DS2-VASc score during the follow-up period was also analyzed by using multiple Cox proportional hazards models.

In Study II, we used chi-square tests to examine the distributions of the categorical data (including age, sex, income status, stroke subtype, history of diseases, and visits to emergency care and inpatient care) among hospitalized stroke patients with and without a history of HF. The adjusted odds ratios (ORs) and 95\% CIs of poststroke pneumonia, septicemia, urinary tract infection, and mortality associated with a history of HF were calculated with multiple logistic regressions. The length of the hospital stay and medical expenditures during stroke admission were also compared between patients with and without HF by using multiple linear regressions and $t$-tests.

\section{Results}

In Study I (the retrospective cohort study), the proportions of patients with low income $(p<0.0001)$, hypertension $(p<0.0001)$, chronic obstructive pulmonary disease $(p<0.0001)$, mental disorders $(p<0.0001)$, diabetes $(p<$ $0.0001)$ atherosclerosis $\quad(p<0.0001), \quad$ hyperlipidemia $(p<0.0001)$, liver cirrhosis $(p<0.0001)$, atrial fibrillation $(p<$ $0.0001)$, renal dialysis $(p<0.0001)$, and Parkinson's disease $(p<0.0001)$ were higher in the HF cohort than in the non-HF cohort (Table 1). Compared with non-HF subjects, patients with $\mathrm{HF}$ were more likely to have experienced hospitalization $(p<0.0001)$ and emergency visits $(p<0.0001)$. The baseline characteristics of people with heart failure by sex were shown in Table S1.

Compared with the non-HF cohort (Table 2), the HF cohort had a higher risk of developing stroke (HR 2.32, 95\% CI 2.21-2.43). The HR for stroke associated with HF was 2.38 (95\% CI 2.22-2.54) in women and 2.26 (95\% CI 2.12-2.41) in men. The association between HF and the risk of stroke was significant in all age groups and in subjects with $0,1,2$, and $\geq 3$ medical conditions. The sensitivity test showed that HF remained significantly associated with stroke risk during the follow-up period when patients who developed stroke in the first 6 months (HR 1.92, 95\% CI 1.83-2.02) and 12 months (HR 1.72, 95\% CI 1.63-1.81) after the onset of HF were excluded (Table 3). The HRs of ischemic stroke, hemorrhagic stroke, and other stroke subtypes associated with HF were 2.69 (95\% CI 2.46-2.94), 2.21 (95\% CI 1.85-2.65), and 2.24 (95\% CI 2.11-2.38), respectively. Lower risks of stroke were found in HF patients using statins (HR 0.84, 95\% CI 0.77-0.91) and metformin (HR 0.59, 95\% CI 
Table I Baseline Characteristics of People with and without Heart Failure Before Follow-Up

\begin{tabular}{|c|c|c|c|c|c|}
\hline \multirow[b]{2}{*}{ Sex } & \multicolumn{2}{|c|}{$\begin{array}{l}\text { No HF } \\
(N=73,492)\end{array}$} & \multicolumn{2}{|c|}{$\begin{array}{l}\text { HF } \\
(N=18,373)\end{array}$} & \multirow{4}{*}{$\begin{array}{l}p \\
1.0000\end{array}$} \\
\hline & $\mathrm{n}$ & (\%) & $\mathrm{n}$ & $(\%)$ & \\
\hline Female & 36,672 & $(49.9)$ & 9168 & $(49.9)$ & \\
\hline Male & 36,820 & $(50.1)$ & 9205 & $(50.1)$ & \\
\hline Age, years & & & & & 1.0000 \\
\hline $30-39$ & 1684 & $(2.3)$ & 421 & (2.3) & \\
\hline $40-49$ & 4480 & $(6.1)$ & 1120 & $(6.1)$ & \\
\hline $50-59$ & 8156 & $(I I .1)$ & 2039 & (II.I) & \\
\hline $60-69$ & 15,080 & $(20.5)$ & 3770 & $(20.5)$ & \\
\hline $70-79$ & 24,880 & $(33.9)$ & 6220 & $(33.9)$ & \\
\hline$\geq 80$ & 19,212 & $(26.1)$ & 4803 & $(26.1)$ & \\
\hline Low income & 2753 & (3.8) & 1254 & $(6.8)$ & $<0.0001$ \\
\hline Medical conditions & & & & & \\
\hline Hypertension & 17,392 & $(23.7)$ & 8759 & $(47.7)$ & $<0.0001$ \\
\hline COPD & 4995 & $(6.8)$ & 4128 & $(22.5)$ & $<0.0001$ \\
\hline Mental disorders & 7438 & $(10.1)$ & 3439 & $(18.7)$ & $<0.0001$ \\
\hline Diabetes & 7322 & $(10.0)$ & 4268 & $(23.2)$ & $<0.0001$ \\
\hline Atherosclerosis & $|75|$ & $(2.4)$ & 1867 & $(10.2)$ & $<0.0001$ \\
\hline Hyperlipidemia & 2804 & (3.8) & 903 & $(4.9)$ & 0.0017 \\
\hline Liver cirrhosis & 1243 & $(1.7)$ & 621 & (3.4) & $<0.0001$ \\
\hline Renal dialysis & 898 & $(1.2)$ & 533 & $(2.9)$ & $<0.0001$ \\
\hline Parkinson's disease & & & & & $<0.0001$ \\
\hline $\begin{array}{l}\text { Number of } \\
\text { hospitalizations }\end{array}$ & 42,634 & $(58.0)$ & 1820 & $(9.9)$ & $<0.0001$ \\
\hline 0 & 11,197 & $(\mid 5.2)$ & 3755 & $(20.4)$ & \\
\hline 1 & 6510 & $(8.9)$ & 2853 & $(15.5)$ & \\
\hline 2 & $|3,15|$ & $(17.9)$ & 9945 & $(54.1)$ & \\
\hline$\geq 3$ & & & & & \\
\hline $\begin{array}{l}\text { Number of } \\
\text { emergency visits }\end{array}$ & 36,314 & $(49.4)$ & 3462 & $(18.8)$ & $<0.0001$ \\
\hline 0 & 11,706 & $(15.9)$ & 3418 & $(18.6)$ & \\
\hline I & 7716 & $(10.5)$ & 2427 & $(13.2)$ & \\
\hline 2 & 17,756 & $(24.2)$ & 9066 & $(49.3)$ & \\
\hline$\geq 3$ & 17,756 & $(24.2)$ & 9066 & $(49.3)$ & \\
\hline Use of anticoagulants & 4235 & $(5.8)$ & 5052 & $(27.5)$ & $<0.0001$ \\
\hline $\begin{array}{l}\text { CHA2DS2-VASc } \\
\text { score* }\end{array}$ & & & & & $<0.0001$ \\
\hline 0 & 12,482 & $(I 7.0)$ & 0 & $(0.0)$ & \\
\hline I & 15,830 & $(21.5)$ & 1512 & $(8.2)$ & \\
\hline 2 & 30,187 & $(4 I .1)$ & 3236 & $(17.6)$ & \\
\hline 3 & 12,364 & $(16.8)$ & 6142 & $(33.4)$ & \\
\hline 4 & 2609 & (3.6) & 5975 & $(32.5)$ & \\
\hline$\geq 5$ & 20 & $(0.0)$ & 1508 & $(8.2)$ & \\
\hline
\end{tabular}

Note: *Time-varying assessments of CHA2DS2-VASc that was calculated at the end of follow-up period.

Abbreviations: COPD, chronic obstructive pulmonary disease; $\mathrm{HF}$, heart failure.
0.51-0.67). Table S2 showed the long-term risks of stroke and all-cause mortality between men and women in people with heart failure. In Table S3, the risk of stroke was associated with CHA2DS2-VASc score in all study subjects. The joint effects of medical conditions on the longterm risk of stroke in patients with and without heart failure are shown in Table S4. HF patients with hypertension had the highest stroke risk compared with non-HF patients without hypertension (HR 5.03, 95\% CI 4.71-5.37).

In Study II (the nested cohort study) of 480,604 patients with stroke hospitalization (Table 4), the group of patients with HF had a higher proportion of females $(p<0.0001)$, people aged $\geq 80$ years $(p<0.0001)$, people with low income $(p=0.0247)$, and people with ischemic stroke $(p<0.0001)$ and were more likely to suffer from comorbid hypertension $(p<0.0001)$, diabetes $(p<0.0001)$, chronic obstructive pulmonary disease $(p<0.0001)$, mental disorders $(p<0.0001)$, atrial fibrillation $(p<0.0001)$, atherosclerosis $(p<0.0001)$, renal dialysis $(p<0.0001)$, liver cirrhosis $(p<0.0001)$, and Parkinson's disease $(p<0.0001)$ compared with those without HF. Higher proportions of patients with previous hospitalizations $(p<0.0001)$ and emergency visits $(p<0.0001)$ were also found in the HF patients than in the non-HF patients. Table S5 showed the characteristics of hospitalized stroke patients with and without heart failure after matching by propensity score.

Compared with non-HF patients (Table 5), HF patients had higher risks of 30-day poststroke pneumonia (OR $1.31,95 \%$ CI $1.24-1.38$ ), septicemia (OR 1.25, 95\% CI 1.17-1.35), intensive care (OR 1.47, 95\% CI 1.41-1.54), and in-hospital mortality (OR 1.44, 95\% CI 1.31-1.59) during or after the index stroke admission. HF patients with stroke admission were also more likely to have increased medical expenditures (beta $=329, p<0.0001$ ) and prolonged hospital stays (beta $=0.78, p<0.0001$ ). Table S6 showed the outcomes of stroke patients with and without history of heart failure after matching by propensity score.

\section{Discussion}

Study I demonstrated that HF independently increased the long-term risk of stroke, and the association was significant in all age groups, both sexes, and in patients with various medical conditions. Study II showed that a history of HF was associated with various outcomes during stroke hospitalization, including poststroke mortality, infections, 
Table 2 Long-Term Risk of Stroke for People with and without Heart Failure in the Overall and Stratified Analysis

\begin{tabular}{|c|c|c|c|c|c|c|}
\hline & $\mathbf{N}$ & Events & Person-Years & Incidence* & HR & $(95 \% \mathrm{Cl}) \dagger$ \\
\hline No heart failure & 73,492 & 6336 & 596,129 & 10.6 & 1.00 & (reference) \\
\hline Heart failure & 18,373 & 3211 & 137,217 & 23.4 & 1.82 & $(1.71-1.93)$ \\
\hline \multicolumn{7}{|l|}{ Female } \\
\hline No heart failure & 36,672 & 3008 & 301,688 & 9.97 & 1.00 & (reference) \\
\hline Heart failure & 9168 & 1598 & 71,072 & 22.5 & 1.91 & $(1.75-2.08)$ \\
\hline \multicolumn{7}{|l|}{ Male } \\
\hline No heart failure & 36,820 & 3328 & $294,44 I$ & 11.3 & 1.00 & (reference) \\
\hline Heart failure & 9205 & 1613 & 66,145 & 24.4 & 1.75 & $(1.61-1.90)$ \\
\hline \multicolumn{7}{|l|}{ Age, $30-49$ years } \\
\hline No heart failure & 6164 & 117 & 57,078 & 2.05 & 1.00 & (reference) \\
\hline Heart failure & $|54|$ & 162 & 13,282 & 12.2 & 2.84 & $(1.95-4.14)$ \\
\hline \multicolumn{7}{|l|}{ Age, $50-59$ years } \\
\hline No heart failure & 8156 & 382 & 70,689 & 5.40 & 1.00 & (reference) \\
\hline Heart failure & 2039 & 302 & 16,335 & 18.5 & 2.70 & $(2.14-3.41)$ \\
\hline \multicolumn{7}{|l|}{ Age, $60-69$ years } \\
\hline No heart failure & 15,080 & $|35|$ & 130,046 & 10.4 & 1.00 & (reference) \\
\hline Heart failure & 3770 & 709 & 30,919 & 22.9 & 2.05 & $(1.81-2.33)$ \\
\hline \multicolumn{7}{|l|}{ Age, $70-79$ years } \\
\hline No heart failure & 24,880 & 3051 & 197,202 & 15.5 & 1.00 & (reference) \\
\hline Heart failure & 6220 & 1285 & 45,153 & 28.5 & 1.92 & $(1.75-2.10)$ \\
\hline \multicolumn{7}{|l|}{ Age, $\geq 80$ years } \\
\hline No heart failure & 19,212 & 1435 & 141,115 & 10.2 & 1.00 & (reference) \\
\hline Heart failure & 4803 & 753 & 31,528 & 23.9 & 1.13 & $(0.98-1.30)$ \\
\hline \multicolumn{7}{|l|}{0 medical condition } \\
\hline No heart failure & 43,364 & 2202 & 361,932 & 6.08 & 1.00 & (reference) \\
\hline Heart failure & $4|5|$ & 589 & 34,035 & 17.3 & 2.05 & $(I .8 I-2.31)$ \\
\hline \multicolumn{7}{|l|}{ I medical condition } \\
\hline No heart failure & $19,4 \mid 4$ & 2413 & 155,028 & 15.6 & 1.00 & (reference) \\
\hline Heart failure & 6904 & 1208 & 52,523 & 23.0 & 1.73 & $(1.59-1.90)$ \\
\hline \multicolumn{7}{|l|}{2 medical conditions } \\
\hline No heart failure & 8012 & 1235 & 60,179 & 20.5 & 1.00 & (reference) \\
\hline Heart failure & 4714 & 874 & 33,841 & 25.8 & 1.48 & $(1.32-1.66)$ \\
\hline \multicolumn{7}{|l|}{$\geq 3$ medical conditions } \\
\hline No heart failure & 2702 & 486 & 18,990 & 25.6 & 1.00 & (reference) \\
\hline Heart failure & 2604 & 540 & 16,817 & 32.1 & 1.62 & $(1.37-1.91)$ \\
\hline
\end{tabular}

Notes: *Per 1000 person-years. †Adjusted for all covariates listed in Tables I and SI.

Abbreviations: $\mathrm{Cl}$, confidence interval; $\mathrm{HR}$, hazard ratio.

admission to intensive care, a prolonged length of stay, and increased medical expenditures.

Compared with previous studies, ${ }^{6-17}$ the present study benefitted from a large sample size, nationwide coverage and complete follow-up for 14 years. The association between HF and long-term stroke risk was consistently significant among patients with or without atrial fibrillation or atherosclerosis. In addition, the stroke risk in HF patients was attenuated but persisted over time ( 6 and 12 months after the onset of HF), which is in agreement with the results of some previous studies. ${ }^{7,12,14}$ However, another study showed that the risk of ischemic stroke was normalized 6 months after the diagnosis of HF. ${ }^{11}$ Furthermore, our analysis showed that HF increased the risk of both ischemic and 
Table 3 Sensitivity Analyses for the Association Between Heart Failure and Stroke Risk

\begin{tabular}{|c|c|c|c|c|c|c|}
\hline & $\mathbf{N}$ & Events & PYs & Incidence* & HR & $(95 \% \mathrm{Cl}) \dagger$ \\
\hline \multicolumn{7}{|l|}{ Excluding stroke cases in 6 months } \\
\hline No HF & 72,984 & 5828 & 596,009 & 9.78 & 1.00 & (reference) \\
\hline $\mathrm{HF}$ & 17,807 & 2645 & 137,105 & 19.3 & 1.50 & $(1.40-1.60)$ \\
\hline \multicolumn{7}{|l|}{ Excluding stroke cases in 12 months } \\
\hline No HF & 72,520 & 5364 & 595,666 & 9.01 & 1.00 & (reference) \\
\hline $\mathrm{HF}$ & 17,439 & 2277 & 136,835 & 16.6 & 1.31 & $(1.22-1.40)$ \\
\hline \multicolumn{7}{|l|}{ Risk of ischemic stroke } \\
\hline No HF & 68,873 & 1717 & 575,942 & 2.98 & 1.00 & (reference) \\
\hline $\mathrm{HF}$ & 16,102 & 940 & 129,835 & 7.24 & 1.95 & $(1.74-2.18)$ \\
\hline \multicolumn{7}{|l|}{ Risk of hemorrhagic stroke } \\
\hline No HF & 67,629 & 473 & 570,560 & 0.83 & 1.00 & (reference) \\
\hline $\mathrm{HF}$ & 15,383 & 221 & 127,458 & 1.73 & 1.91 & $(1.52-2.40)$ \\
\hline \multicolumn{7}{|l|}{ Risk of other stroke subtypes } \\
\hline No HF & 71,302 & 4146 & 586,824 & 7.07 & 1.00 & (reference) \\
\hline $\mathrm{HF}$ & 17,212 & 2050 & 133,663 & 15.3 & 1.77 & $(1.64-1.91)$ \\
\hline Non-HF cohort & 73,492 & 6336 & 596,129 & 10.6 & 1.00 & (reference) \\
\hline \multicolumn{7}{|l|}{ HF cohort } \\
\hline injected diuretics & 12,570 & 1788 & 93,662 & 19.1 & 1.47 & $(1.37-1.58)$ \\
\hline injected cardiac stimulant & 8202 & 649 & 60,892 & 10.7 & 0.80 & $(0.73-0.88)$ \\
\hline HF patients without use of statins & 12,995 & 2333 & 93,600 & 24.9 & 1.00 & (reference) \\
\hline HF patients with use of statins & 5378 & 878 & 43,617 & 20.1 & 0.85 & $(0.78-0.93)$ \\
\hline HF patients without use of metformin & 16,426 & 2953 & 120,675 & 24.5 & 1.00 & (reference) \\
\hline HF patients with use of metformin & 1947 & 258 & 16,542 & 15.6 & 0.64 & $(0.56-0.73)$ \\
\hline
\end{tabular}

Notes: Sensitivity test: HF was significantly associated with stroke risk after excluding cases developing stroke in the first 6 and 12 months after the onset of HF. *Per 1000 person-years. †Adjusted for all covariates listed in Tables I and SI.

hemorrhagic stroke. Two studies have also reported an elevated risk of hemorrhagic stroke in HF patients, ${ }^{12,14}$ while the Rotterdam Study found a reduced risk of hemorrhagic stroke in HF patients compared to that in the general population. ${ }^{11}$ The lower risk of stroke in HF patients treated with intravenous diuretics and cardiac stimulants during admission is also of interest. The conditions that necessitated intravenous diuretics and chronotropic or inotropic agents were most likely acute decompensated HF or advanced HF. ${ }^{20}$ HF-related comorbid diseases may override the effect of HF itself on stroke risk in these patients. Substudies of large multicenter randomized trials have reported that the left ventricular ejection fraction was not an independent predictor for stroke in HF patients without atrial fibrillation. ${ }^{13,17}$ Additionally, our results showed that HF patients using statins and metformin had a reduced risk of stroke, which has important implications for stroke prevention in HF. A meta-analysis of randomized trials showed that each $1 \mathrm{mmol} \cdot \mathrm{L}^{-1}$ decrease in $\mathrm{LDL}$ cholesterol lowers stroke risk by $21.1 \%,{ }^{21}$ which is in line with our results. Additionally, metformin has been shown to attenuate diabetes-accelerated atherosclerosis by inhibiting mitochondrial fission in endothelial cells and protecting against coronary atherosclerosis in prediabetes and early diabetes. ${ }^{22,23}$ Our epidemiologic analysis warrants further clinical trials to prove the effectiveness of metformin in the treatment of vascular atherosclerosis.

Several possible mechanisms may be helpful for explaining the increased risk of stroke in patients with HF. First, with respect to ischemic stroke, the stasis of blood flow in the dilated and hypokinetic ventricle may facilitate thrombus formation. ${ }^{24}$ Therefore, to examine whether HF itself causes ischemic stroke, it is necessary to evaluate the risk of ischemic stroke in HF patients without atrial fibrillation or systolic abnormalities. Second, HF patients are in a hypercoagulable state, which is characterized by platelet hyperactivity, impaired fibrinolysis, and greater thrombin generation. ${ }^{25}$ The 
Table 4 Characteristics of Hospitalized Stroke Patients with and without Heart Failure

\begin{tabular}{|c|c|c|c|c|c|}
\hline \multirow[b]{2}{*}{ Sex } & \multicolumn{2}{|c|}{$\begin{array}{l}\text { No HF } \\
(N=450,072)\end{array}$} & \multicolumn{2}{|c|}{$\begin{array}{l}H F \\
(N=30,532)\end{array}$} & \multirow{4}{*}{$\begin{array}{l}\mathbf{p} \\
<0.0001\end{array}$} \\
\hline & $n$ & (\%) & $\mathrm{n}$ & (\%) & \\
\hline Female & $|84,4| \mid$ & $(41.0)$ & 15,406 & $(50.5)$ & \\
\hline Male & 265,661 & $(59.0)$ & 15,126 & (49.5) & \\
\hline Age, years & & & & & $<0.0001$ \\
\hline $30-39$ & 10,885 & $(2.4)$ & 196 & $(0.6)$ & \\
\hline $40-49$ & 39,164 & $(8.7)$ & 963 & $(3.2)$ & \\
\hline $50-59$ & 77,096 & (17.1) & 2388 & (7.8) & \\
\hline $60-69$ & 110,684 & (24.6) & 5503 & $(18.0)$ & \\
\hline 70-79 & 138,016 & (30.7) & 11,565 & (37.9) & \\
\hline$\geq 80$ & 74,227 & $(16.5)$ & 9917 & $(32.5)$ & \\
\hline Low income & 27,059 & $(6.0)$ & 2241 & $(7.3)$ & $<0.0001$ \\
\hline Medical conditions & & & & & \\
\hline Hypertension & 210,270 & $(46.7)$ & 17,770 & $(58.2)$ & $<0.0001$ \\
\hline Diabetes & 104,825 & (23.3) & 9131 & $(29.9)$ & $<0.0001$ \\
\hline Hyperlipidemia & 19,074 & $(4.2)$ & 1273 & $(4.2)$ & 0.5646 \\
\hline Atherosclerosis & 9800 & $(2.2)$ & 2160 & (7.1) & $<0.0001$ \\
\hline Atrial fibrillation & 5438 & $(1.2)$ & 2620 & $(8.6)$ & $<0.0001$ \\
\hline COPD & 43,329 & $(9.6)$ & 8509 & (27.9) & $<0.0001$ \\
\hline Liver cirrhosis & $|I, 01|$ & $(2.5)$ & 979 & $(3.2)$ & $<0.0001$ \\
\hline Renal dialysis & 7466 & $(1.7)$ & 1830 & $(6.0)$ & $<0.0001$ \\
\hline Parkinson's disease & 8345 & $(1.9)$ & 806 & $(2.6)$ & $<0.0001$ \\
\hline Mental disorders & 74,150 & $(16.5)$ & 6933 & (22.7) & $<0.0001$ \\
\hline Type of stroke & & & & & $<0.0001$ \\
\hline $\begin{array}{l}\text { Hemorrhagic } \\
\text { stroke }\end{array}$ & 111,603 & (24.8) & 4798 & (15.7) & \\
\hline Ischemic stroke & 281,676 & (62.6) & 21,235 & (69.6) & \\
\hline Other stroke & 56,793 & $(12.6)$ & 4499 & (14.7) & \\
\hline $\begin{array}{l}\text { Number of } \\
\text { hospitalizations }\end{array}$ & & & & & $<0.0001$ \\
\hline 0 & 343,022 & $(76.2)$ & 10,479 & $(34.3)$ & \\
\hline I & 67,635 & $(15.0)$ & 8521 & (27.9) & \\
\hline 2 & 21,862 & $(4.9)$ & 5057 & $(16.6)$ & \\
\hline$\geq 3$ & 17,553 & (3.9) & 6475 & $(21.2)$ & \\
\hline $\begin{array}{l}\text { Number of emergency } \\
\text { visits }\end{array}$ & & & & & $<0.0001$ \\
\hline 0 & 266,457 & (59.2) & 10,752 & $(35.2)$ & \\
\hline I & 109,655 & (24.4) & 7640 & $(25.0)$ & \\
\hline 2 & 41,143 & $(9.1)$ & 4794 & (15.7) & \\
\hline$\geq 3$ & 32,817 & $(7.3)$ & 7346 & (24.1) & \\
\hline Use of antibiotics* & 76,480 & $(17.0)$ & 8860 & $(29.0)$ & $<0.0001$ \\
\hline
\end{tabular}

Note: *Use of antibiotics within 3 months before stroke admission.

Abbreviations: COPD, chronic obstructive pulmonary disease; HF, heart failure.

activation of the renin-angiotensin-aldosterone system and the sympathetic nervous system in HF further aggravates the prothrombotic state. ${ }^{26}$ Third, the decreased cerebral blood flow in HF patients results from low cardiac output and impaired cerebral autoregulation. ${ }^{27}$ Diminished cerebral perfusion may increase the risk of cognitive impairment among both decompensated and stable HF patients. ${ }^{27}$ Finally, HF patients are prone to have largeartery atherosclerosis and small-vessel occlusion, which also contributes to the development of ischemic stroke. ${ }^{28}$ In HF patients with sinus rhythm, the mechanism of ischemic stroke was shown to be predominantly influenced by the etiology of HF, and HF due to coronary artery disease was associated with the atherosclerotic and lacunar subtypes of stroke. $^{28}$ Regarding hemorrhagic stroke, antiplatelet or anticoagulant agents administered in HF patients may increase the risk of hemorrhage and cause intracranial bleeding. ${ }^{29}$

Our analysis showed that the impact of HF on the occurrence of stroke was augmented in younger patients compared with older patients. Studies have shown that idiopathic dilated cardiomyopathy accounts for a substantial part of the etiology of early-onset HF. ${ }^{30,31}$ The atrial and ventricular dilation in cardiomyopathy and lower adherence to medication may underlie the greater influence of $\mathrm{HF}$ on stroke risk in young adults compared with older individuals. ${ }^{30}$ However, it is still controversial whether anticoagulation has a favorable risk-benefit profile when used for primary thromboembolism prophylaxis in patients with HF secondary to dilated cardiomyopathy with sinus rhythm. ${ }^{32}$ Currently, nonvitamin K oral anticoagulants have been broadly used for stroke prophylaxis in atrial fibrillation patients. ${ }^{33}$ The determination of the benefits and risks of nonvitamin $\mathrm{K}$ oral anticoagulants for stroke prevention in HF patients with sinus rhythm awaits further investigation.

Compared with patients without HF, patients with HF had worse poststroke outcomes according to our results, which is in accordance with the results of prior studies. ${ }^{12,34,35} \mathrm{In}$ addition to the mortality rate, the risk of pneumonia, septicemia, and urinary tract infection was also increased in patients with HF. Studies have indicated that stroke induces systemic immunosuppression and increases the risk of infection. ${ }^{36}$ Infections commonly occur after stroke with a reported incidence of approximately $30 \%$ and cause significant mortality and morbidity in stroke patients. ${ }^{37}$ Similarly, impaired immune responses occur in HF, including elevated levels of serum tumor necrosis factor, interleukin-1, interleukin-6 and interleukin-10. ${ }^{38,39}$ Recently, the impairment of cellmediated immune functioning was reported to predict longterm mortality in patients with advanced $\mathrm{HF}^{40}$ It is still controversial whether the immune and inflammation response is a cause or a consequence of myocardial failure. 
Table 5 Outcomes of Stroke Patients with and without History of Heart Failure

\begin{tabular}{|c|c|c|c|c|c|c|}
\hline \multirow[t]{2}{*}{ Poststroke Outcomes } & \multicolumn{2}{|c|}{ No HF $(N=450,072)$} & \multicolumn{2}{|c|}{$H F(N=30,532)$} & \multicolumn{2}{|c|}{ Risk of Outcomes } \\
\hline & Events & (\%) & Events & $(\%)$ & OR & $(95 \% \mathrm{Cl}) \dagger$ \\
\hline Mortality & 13,064 & 2.9 & 1267 & 4.2 & 1.40 & $(1.31-1.50)$ \\
\hline Pneumonia & 38,408 & 8.5 & 3951 & 12.9 & 1.33 & $(1.28-1.38)$ \\
\hline Septicemia & 17,212 & 3.8 & 2119 & 6.9 & 1.31 & $(1.24-1.38)$ \\
\hline UTI & 50,222 & 11.3 & 4249 & 13.9 & 1.02 & $(0.98-1.05)$ \\
\hline ICU stay & 92,062 & 20.5 & 7360 & 24.1 & 1.52 & $(1.47-1.57)$ \\
\hline Medical expenditure, USD* & \multicolumn{2}{|c|}{$2232 \pm 3470$} & \multicolumn{2}{|c|}{$2569 \pm 3585$} & \multicolumn{2}{|c|}{$p<0.0001$} \\
\hline Length of hospital stay, day* & \multicolumn{2}{|c|}{$11.6 \pm 13.4$} & \multicolumn{2}{|c|}{$13.2 \pm 13.7$} & \multicolumn{2}{|c|}{$p<0.000 I$} \\
\hline
\end{tabular}

Notes: *Mean \pm SD; HF was associated with medical expenditure (beta $=33 \mathrm{I}, p<0.000 \mathrm{I})$ and length of hospital stay $($ beta $=0.79, p<0.000 \mathrm{I})$ in the multiple linear regressions. †Adjusted for all covariates listed in Table 4.

Abbreviations: $\mathrm{Cl}$, confidence interval; $\mathrm{HF}$, heart failure; ICU, intensive care unit; OR, odds ratio; UTI, urinary tract infection.

Furthermore, the interaction between HF and stroke and the combined effect on the immune system remain to be observed.

Our findings highlight the need to develop strategies of reducing stroke risk and poststroke complications for HF population. Apart from warfarin, nonvitamin K oral anticoagulants have been broadly used for primary thromboembolism prophylaxis in patients with atrial fibrillation. ${ }^{33}$ For HF patients with sinus rhythm, anticoagulation using warfarin reduces the risk of non-fatal stroke but increases the risk of major hemorrhage. ${ }^{32}$ Recent trial showed that an addition of rivaroxaban to antiplatelet therapy significantly reduces the rate of first stroke with similar hemorrhage risk among HF patients with sinus rhythm. ${ }^{41}$ The benefits and risks of nonvitamin $\mathrm{K}$ oral anticoagulants for stroke prophylaxis in $\mathrm{HF}$ awaits further evaluations. Regarding the prevention of poststroke infection (e.g. pneumonia), both pharmacological and non-pharmacological approaches have been studied extensively. Meta-analysis showed that prophylactic antibiotics initiated within 48 hours after acute stroke reduce the risk of poststroke pneumonia and all infections, but functional outcomes and mortality are not decreased. ${ }^{42}$ There is still insufficient evidence supporting routine uses of prophylactic antibiotics for prevention of poststroke infection; the benefits and harms remain undetermined. ${ }^{42,43}$ In addition to antibiotics, angiotensin-converting enzyme inhibitors appear effective in reducing the risk of poststroke pneumonia compared with other antihypertensive agents, especially in Asian populations. ${ }^{44}$ Non-pharmacological prevention of poststroke pneumonia includes swallowing rehabilitation and oral care. ${ }^{45}$

Some limitations need to be acknowledged when interpreting the results. First, our data lacks information about socioeconomics, lifestyle, biochemical laboratory measures, and clinical risk scores of HF and stroke (such as those based on the New York Heart Association functional class symptoms and the NIH Stroke Scale). Second, although the administration of intravenous diuretics and cardiac stimulants could reflect the severity of HF in part, our analysis did not include measurement of left ventricular ejection fraction due to data unavailability, precluding a stratified analysis of stroke risk in HF with reduced, mid-range or preserved left ventricular ejection fraction. In addition, our study did not include people with subclinical HF because they may not seek conventional medical care. Finally, the residual confounding bias is possible, although our analysis has been adjusted for various potential confounders.

In conclusion, patients with HF had increased stroke risk and poststroke adverse outcomes. We propose that there is an urgent need for intervention to prevent stroke events and poststroke complications in this susceptible population.

\section{Abbreviations}

ICD-9-CM, International Classification of Diseases, 9th Revision, Clinical Modification; CI, confidence interval; $\mathrm{HF}$, heart failure; HR, hazard ratio; OR, odds ratio.

\section{Author Contributions}

YHT, CCL: conception and design, analysis and interpretation of the data, drafting the article, agree on the journal to which the article will be submitted, take responsibility and be accountable for the contents of the article, critical revision of the manuscript for important intellectual content and final approval of the version to be published. CCC, CCY, LCS, CJH, YGC, TLC: conception and design, interpretation of the data, agree on the journal to 
which the article will be submitted, take responsibility and be accountable for the contents of the article, critical revision of the manuscript for important intellectual content and final approval of the version to be published. All authors have read and approved the submitted manuscript.

\section{Funding}

This work was supported in part by The Higher Education Sprout Project by the Ministry of Edication (DP2-10921121-01-N-08-04) and Taiwan's Ministry of Science and Technology (MOST106-2314-B-038-036-MY3; MOST107-2221-E-038-009).

\section{Disclosure}

Yih-Giun Cherng and Chien-Chang Liao are corresponding author for this study. Ying-Hsuan Tai and Ta-Liang Chen are co-first authors for this study. The authors report no conflicts of interest in this work.

\section{References}

1. GBD. 2016 stroke collaborators. Global, regional, and national burden of stroke, 1990-2016: a systematic analysis for the global burden of disease study 2016. Lancet Neurol. 2019;18(5):439-458.

2. Boehme AK, Esenwa C, Elkind MSV. Stroke risk factors, genetics, and prevention. Circ Res. 2017;120(3):472-495. doi:10.1161/ CIRCRESAHA. 116.308398

3. GBD. 2017 disease and injury incidence and prevalence collaborators. Global, regional, and national incidence, prevalence, and years lived with disability for 354 diseases and injuries for 195 countries and territories, 1990-2017: a systematic analysis for the global burden of disease study 2017. Lancet. 2018;392(10159):1789-1858.

4. Cook C, Cole G, Asaria P, et al. The annual global economic burden of heart failure. Int J Cardiol. 2014;171(3):368-376. doi:10.1016/j. ijcard.2013.12.028

5. Kim W, Kim EJ. Heart failure as a risk factor for stroke. J Stroke. 2018;20(1):33-45. doi:10.5853/jos.2017.02810

6. Dries DL, Rosenberg YD, Waclawiw MA, et al. Ejection fraction and risk of thromboembolic events in patients with systolic dysfunction and sinus rhythm: evidence for gender differences in the studies of left ventricular dysfunction trials. J Am Coll Cardiol. 1997;29 (5):1074-1080. doi:10.1016/S0735-1097(97)00019-3

7. Witt BJ, Brown RD Jr, Jacobsen SJ, et al. Ischemic stroke after heart failure: a community-based study. Am Heart J. 2006;152(1):102-109. doi:10.1016/j.ahj.2005.10.018

8. Hays AG, Sacco RL, Rundek T, et al. Left ventricular systolic dysfunction and the risk of ischemic stroke in a multiethnic population. Stroke. 2006;37(7):1715-1719. doi:10.1161/01. STR.0000227121.34717.40

9. Freudenberger RS, Hellkamp AS, Halperin JL, et al. Risk of thromboembolism in heart failure: an analysis from the Sudden Cardiac Death in Heart Failure Trial (SCD-HeFT). Circulation. 2007;115 (20):2637-2641. doi:10.1161/CIRCULATIONAHA.106.661397

10. Pullicino PM, McClure LA, Wadley VG, et al. Blood pressure and stroke in heart failure in the Reasons for Geographic And Racial Differences in Stroke (REGARDS) study. Stroke. 2009;40 (12):3706-3710. doi:10.1161/STROKEAHA.109.561670
11. Alberts VP, Bos MJ, Koudstaal P, et al. Heart failure and the risk of stroke: the Rotterdam Study. Eur J Epidemiol. 2010;25(11):807-812. doi:10.1007/s10654-010-9520-y

12. Lip GYH, Rasmussen LH, Skjøth F, et al. Stroke and mortality in patients with incident heart failure: the Diet, Cancer and Health (DCH) cohort study. BMJ Open. 2012;2(4):e000975. doi:10.1136/ bmjopen-2012-000975

13. Abdul-Rahim AH, Perez AC, Fulton RL, et al. Risk of stroke in chronic heart failure patients without atrial fibrillation: analysis of the Controlled Rosuvastatin in Multinational Trial Heart Failure (CORONA) and the Gruppo Italiano per lo Studio della Sopravvivenza nell'Insufficienza Cardiaca-Heart Failure (GISSI-HF) Trials. Circulation. 2015;131(17):1486-1494.

14. Adelborg K, Szépligeti S, Sundbøll J, et al. Risk of stroke in patients with heart failure: a population-based 30-year cohort study. Stroke. 2017;48(5):1161-1168. doi:10.1161/STROKEAHA.116.016022

15. Kang S-H, Kim J, Park JJ, et al. Risk of stroke in congestive heart failure with and without atrial fibrillation. Int $J$ Cardiol. 2017;248:182-187. doi:10.1016/j.ijcard.2017.07.056

16. Mogensen UM, Jhund PS, Abraham WT, et al. Type of atrial fibrillation and outcomes in patients with heart failure and reduced ejection fraction. J Am Coll Cardiol. 2017;70(20):2490-2500. doi:10.1016/j. jacc.2017.09.027

17. Abdul-Rahim AH, Perez AC, MacIsaac RL, et al. Risk of stroke in chronic heart failure patients with preserved ejection fraction, but without atrial fibrillation: analysis of the CHARM-preserved and I-preserve trials. Eur Heart J. 2017;38(10):742-750.

18. Liao -C-C, Chou Y-C, Yeh C-C, et al. Stroke risk and outcomes in patients with traumatic brain injury: 2 nationwide studies. Mayo Clin Proc. 2014;89(2):163-172. doi:10.1016/j.mayocp.2013.09.019

19. Liao CC, Chang PY, Yeh CC, et al. Outcomes after surgery in patients with previous stroke. Br J Surg. 2014;101(12):1616-1622. doi:10.1002/bjs. 9639

20. Crespo-Leiro MG, Metra M, Lund LH, et al. Advanced heart failure: a position statement of the Heart Failure Association of the European Society of Cardiology. Eur J Heart Fail. 2018;20(11):1505-1535.

21. Amarenco P, Labreuche J. Lipid management in the prevention of stroke: review and updated meta-analysis of statins for stroke prevention. Lancet Neurol. 2009;8(5):453-463. doi:10.1016/S14744422(09)70058-4

22. Wang Q, Zhang M, Torres G, et al. Metformin suppresses diabetes-accelerated atherosclerosis via the inhibition of Drp1-mediated mitochondrial fission. Diabetes. 2017;66 (1):193-205. doi:10.2337/db16-0915

23. Goldberg RB, Aroda VR, Bluemke DA, et al. Effect of long-term metformin and lifestyle in the diabetes prevention program and its outcome study on coronary artery calcium. Circulation. 2017;136 (1):52-64. doi:10.1161/CIRCULATIONAHA.116.025483

24. Di Tullio MR, Qian M, Thompson JLP, et al. Left ventricular ejection fraction and risk of stroke and cardiac events in heart failure: data from the warfarin versus aspirin in reduced ejection fraction trial. Stroke. 2016;47(8):2031-2037. doi:10.1161/STROKEAHA.116.01 3679

25. Shantsila E, Lip GYH. Thrombotic complications in heart failure: an underappreciated challenge. Circulation. 2014;130(5):387-389. doi:10.1161/CIRCULATIONAHA.114.011353

26. Verbrugge FH, Tang WH, Mullens W. Renin-angiotensin-aldosterone system activation during decongestion in acute heart failure: friend or foe? JACC Heart Fail. 2015;3(2):108-111. doi:10.1016/j. jchf.2014.10.005

27. Havakuk O, King KS, Grazette L, et al. Heart failure-induced brain injury. J Am Coll Cardiol. 2017;69(12):1609-1616. doi:10.1016/j. jacc.2017.01.022

28. Vemmos K, Ntaios G, Savvari P, et al. Stroke aetiology and predictors of outcome in patients with heart failure and acute stroke: a 10-year follow-up study. Eur J Heart Fail. 2012;14(2):211-218. 
29. Melkonian M, Jarzebowski W, Pautas E, et al. Bleeding risk of antiplatelet drugs compared with oral anticoagulants in older patients with atrial fibrillation: a systematic review and meta-analysis. J Thromb Haemost. 2017;15(7):1500-1510. doi:10.1111/jth.13697

30. Barasa A, Schaufelberger M, Lappas G, et al. Heart failure in young adults: 20-year trends in hospitalization, aetiology, and case fatality in Sweden. Eur Heart J. 2014;35(1):25-32. doi:10.1093/eurheartj/ eht 278

31. Wong CM, Hawkins NM, Jhund PS, et al. Clinical characteristics and outcomes of young and very young adults with heart failure: the CHARM programme (Candesartan in Heart Failure Assessment of Reduction in Mortality and Morbidity). J Am Coll Cardiol. 2013;62 (20):1845-1854. doi:10.1016/j.jacc.2013.05.072

32. Beggs SAS, Rørth R, Gardner RS, et al. Anticoagulation therapy in heart failure and sinus rhythm: a systematic review and meta-analysis. Heart. 2019;105(17):1325-1334. doi:10.1136/ heartjnl-2018-314381

33. Shpak M, Ramakrishnan A, Nadasdy Z, et al. Higher incidence of ischemic stroke in patients taking novel oral anticoagulants. Stroke. 2018;49(12):2851-2856. doi:10.1161/STROKEAHA.118.0 22636

34. Appelros P, Nydevik I, Viitanen M. Poor outcome after first-ever stroke: predictors for death, dependency, and recurrent stroke within the first year. Stroke. 2003;34(1):122-126. doi:10.1161/01.STR. $0000047852.05842 .3 \mathrm{C}$

35. Divani AA, Vazquez G, Asadollahi M, et al. Nationwide frequency and association of heart failure on stroke outcomes in the United States. J Card Fail. 2009;15(1):11-16. doi:10.1016/j.cardfail.2008. 09.001

36. Shi K, Wood K, Shi FD, et al. Stroke-induced immunosuppression and poststroke infection. Stroke Vasc Neurol. 2018;3(1):34-41.

37. Giede-Jeppe A, Bobinger T, Gerner ST, et al. Lymphocytopenia is an independent predictor of unfavorable functional outcome in spontaneous intracerebral hemorrhage. Stroke. 2016;47(5):1239-1246. doi:10.1161/STROKEAHA.116.013003
38. Feldman AM, Combes A, Wagner D, et al. The role of tumor necrosis factor in the pathophysiology of heart failure. $\mathrm{J} \mathrm{Am} \mathrm{Coll} \mathrm{Cardiol.}$ 2000;35(3):537-544. doi:10.1016/S0735-1097(99)00600-2

39. Kalogeropoulos A, Georgiopoulou V, Psaty BM, et al. Inflammatory markers and incident heart failure risk in older adults: the health $\mathrm{ABC}$ (health, aging, and body composition) study. J Am Coll Cardiol. 2010;55(19):2129-2137. doi:10.1016/j.jacc.2009.12.045

40. Nagarajan V, Hernandez AV, Cauthen CA, et al. Usefulness of cell-mediated immune function in risk stratification for patients with advanced heart failure. Am Heart J. 2017;183:35-39. doi:10.1016/j.ahj.2016.09.008

41. Mehra MR, Vaduganathan M, Fu M, et al. A comprehensive analysis of the effects of rivaroxaban on stroke or transient ischaemic attack in patients with heart failure, coronary artery disease, and sinus rhythm: the COMMANDER HF trial. Eur Heart J. 2019;40(44):3593-3602. doi:10.1093/eurheartj/ehz427

42. Badve MS, Zhou Z, Anderson CS, et al. Effectiveness and safety of antibiotics for preventing pneumonia and improving outcome after acute stroke: systematic review and meta-analysis. J Stroke Cerebrovasc Dis. 2018;27(11):3137-3147. doi:10.1016/j.jstrokecerebrovasdis.2018.07.001

43. Kalra L, Irshad S, Hodsoll J, et al. Prophylactic antibiotics after acute stroke for reducing pneumonia in patients with dysphagia (STROKE-INF): a prospective, cluster-randomised, open-label, masked endpoint, controlled clinical trial. Lancet. 2015;386 (10006):1835-1844. doi:10.1016/S0140-6736(15)00126-9

44. Shinohara Y, Origasa H. Post-stroke pneumonia prevention by angiotensin-converting enzyme inhibitors: results of a meta-analysis of five studies in Asians. Adv Ther. 2012;29(10):900-912. doi:10.1007/s12325-012-0049-1

45. Bath PM, Lee HS, Everton LF. Swallowing therapy for dysphagia in acute and subacute stroke. Cochrane Database Syst Rev. 2018;10 (10):CD000323
Clinical Epidemiology

\section{Publish your work in this journal}

Clinical Epidemiology is an international, peer-reviewed, open access, online journal focusing on disease and drug epidemiology, identification of risk factors and screening procedures to develop optimal preventative initiatives and programs. Specific topics include: diagnosis, prognosis, treatment, screening, prevention, risk factor modification,

\section{Dovepress}

systematic reviews, risk \& safety of medical interventions, epidemiology \& biostatistical methods, and evaluation of guidelines, translational medicine, health policies \& economic evaluations. The manuscript management system is completely online and includes a very quick and fair peer-review system, which is all easy to use. 